Reiner Diedrichs und Ralf Goebel

\title{
Veränderungen sind nötig. Kooperation des Bibliotheksservice-Zentrums Baden-Württemberg (BSZ) und der Verbundzentrale des GBV (VZG)
}

Die Deutsche Forschungsgemeinschaft (DFG), der Wissenschaftsrat (WR) und auch der Rat für Informationsinfrastrukturen (RfII) haben in den vergangenen Jahren in einer Vielzahl von Publikationen die Notwendigkeit der Restrukturierung und der Neuausrichtung der wissenschaftlichen Informationsinfrastrukturen beschrieben. Gefordert wurden nachhaltige Konsolidierungsprozesse sowie der Aufbau neuer und am Bedarf der Forschung orientierter Strukturen. Die angemahnten Strukturreformen betrafen neben einer Vielzahl unterschiedlicher Themenbereiche die deutschen Verbundzentralen, welche seit den späten 1980er Jahren die wesentlichen Strukturen der Informations- und Literaturversorgung für wissenschaftliche Bibliotheken in Deutschland prägen.

Bereits bei ihrer Einrichtungs- und der späteren Etablierungsphase wurden die Verbundzentralen von der DFG begleitet und ihre Ausgestaltung durch Stellungnahmen, Analysen und Fördermaßnahmen unterstützt. Die DFG ist ein wichtiger Bestandteil der Gründungsgeschichte und der Legitimation der Verbundzentralen in Deutschland. Vor diesem Hintergrund haben die Verbundzentralen die Kritik und die Empfehlungen der DFG zur Neuausrichtung der Informationsinfrastrukturen aufmerksam aufgenommen und entsprechende Aktivitäten entwickelt.

\section{Welche Aufgaben haben Verbundzentralen?}

In Deutschland gibt es sechs Verbundzentralen, die sich - bei vielen Gemeinsamkeiten - dennoch hinsichtlich ihrer Größe, ihrer Aufgaben, ihrer Ressourcen, der eingesetzten Verbundsoftware und ihrer institutionellen Struktur erheblich unterscheiden. Während die Verbundzentralen in Bayern, Berlin und Hessen organisatorische Einheiten oder Abteilungen größerer Einrichtungen (Staatsbibliothek, Universität oder Forschungsinstitut) darstellen, sind die Verbundzentralen in Baden-Württemberg, Niedersachsen und Nordrhein-Westfalen Einrichtungen in der Dienst- und Fachaufsicht der jeweiligen Wissenschafts- 
ministerien ihrer Sitzländer. Auch bei der eingesetzten Basissoftware für die Verbundsysteme gibt es Unterschiede: Während ein Teil der Verbundzentralen für ihre Verbunddatenbanken die Software Aleph der Firma Ex Libris einsetzt, nutzt der andere Teil das Central Bibliographic System (CBS) der Firma OCLC.

Neben dem Betrieb der zentralen Verbundkataloge, dem Management von nationalen und internationalen Metadaten (Aggregator-Funktion) und der deutschen Onlinefernleihe ${ }^{1}$ unterstützen die Verbundzentralen eine Vielzahl von Services und Vorhaben aus dem Bibliotheks- und Wissenschaftsbereich: Hosting und Betrieb von lokalen Bibliothekssystemen, Repositorien, Discovery-Services, Mitarbeit an Forschungsprojekten im Bereich Datenmanagement, Projekte zum Aufbau nationaler Strukturen wie die Altkartendatenbank (IKAR) oder die Verzeichnisse deutscher Drucke (VD16, VD17, VD18) im Rahmen der verteilten Nationalbibliothek, Hosting von Nationallizenzen, Aufbau von Plattformen im Bereich Text- und Datamining, Unterstützung von Museen und Archiven und vieles mehr. Sie tragen maßgeblich zum Erfolg und zum nachhaltigen Betrieb nationaler Strukturen wie z. B. der Gemeinsamen Normdatei (GND) oder der nationalen Zeitschriftendatenbank (ZDB) bei. Als Mitglieder im Standardisierungsausschuss an der Deutschen Nationalbibliothek übernehmen sie Verantwortung für die Aufrechterhaltung und die Weiterentwicklung von Standards und Anwendungsbestimmungen.

International wird Deutschland um den guten Organisationsgrad und die effiziente Nutzung seiner Ressourcen durch die zentrale Lokalisierung von Services an wenigen Verbundzentralen beneidet. Insbesondere in den USA beginnen sich erst in den letzten Jahren neben dem WorldCat von OCLC verbundähnliche Strukturen zu etablieren, in deren Rahmen Daten und Services von Bibliotheken gemeinschaftlich aufgebaut, genutzt und für Services im Forschungsumfeld eingesetzt werden.

\section{Veränderung}

Als Reaktion auf die Aufforderung von DFG und WR taten sich zunächst die institutionell nicht eigenständigen Verbundzentralen aus Bayern, Berlin und Hessen zusammen, um im Rahmen eines DFG-geförderten Vorhabens mit dem Namen CIB (Cloudbasierte Infrastruktur für Bibliotheksdaten) als eines von

1 Diese Aufgabe wurde den Verbundzentralen in einem Beschluss der Kultusministerkonferenz mit dem Titel „Die Ordnung des Leihverkehrs in der Bundesrepublik Deutschland. Leihverkehrsordnung“ (LVO) am 19. September 2003 bzw. in aktualisierter Fassung am 10. Oktober 2008 zugewiesen. 
mehreren Zielen die Abschaffung der deutschen Verbundkataloge und die Auslagerung der Katalogisierungsfunktionalitäten an den von OCLC betriebenen WorldCat zu evaluieren. Obwohl viele Ziele aus dem CIB-Projekt erreicht wurden, konnte die deutschlandweite Primärkatalogisierung im WorldCat und damit der Einstieg in die Abschaffung der Verbunddatenbanken nicht einmal prototypisch realisiert werden. Da dies früh absehbar war, beschlossen die beiden Verbundzentralen in Konstanz ${ }^{2}$ und in Göttingen ${ }^{3}$ eine strategische Partnerschaft mit der Absicht, die von der DFG und dem WR benannten Ziele zumindest für zehn deutsche Bundesländer und die Stiftung Preußischer Kulturbesitz durch Modernisierung und Neustrukturierung ihrer Verbundsysteme umzusetzen. ${ }^{4}$ Schließlich entwickelte auch die Arbeitsgemeinschaft der Verbundsysteme (AGV), in der die Verbundzentralen der Bibliotheksverbünde in Deutschland, Österreich und der deutschsprachigen Schweiz sowie die Deutsche Nationalbibliothek und die Zeitschriftendatenbank vertreten sind, eine pragmatisch ausgerichtete Strategie. Gemeinsam stellte die AGV im Herbst 2017 im Rahmen der Sitzung der Sektion 4 des Deutschen Bibliotheksverbandes (dbv) erste Schritte vor, wie die deutschen Verbünde auf die vorgebrachten Veränderungsbedarfe eingehen und wie sie ihre Rolle im Prozess der Neugestaltung der Informationsinfrastrukturen definieren möchten.

\section{Kooperation zwischen dem BSZ und der Verbundzentrale des GBV (VZG)}

Die Kooperation zwischen dem Bibliotheksservice-Zentrum Baden-Württemberg (BSZ) und der Verbundzentrale des GBV (VZG) und die mit ihr einhergehenden die föderalen Grenzen überschreitenden - Strukturveränderungen haben Vorbildcharakter auf dem Weg der Neuausrichtung der Informationsinfrastrukturen. Beide Verbundzentralen zählen zu den größten in Deutschland. Gemeinsam betreuen sie Bibliotheksdaten der Mehrzahl der deutschen staatlichen Universitäten und Hochschulen sowie Staats- und Landesbibliotheken. Eine Vielzahl renommierter Forschungseinrichtungen wie Leibniz-Institute, Fraunhofer-Institute, Helmholtz-Zentren, Max-Planck-Institute, Spezialbibliotheken sowie starke Kultur- und Forschungseinrichtungen werden im Bereich bibliothekarischer und wissenschaftlicher Metadaten durch das BSZ und die VZG ver-

2 Bibliotheksservice-Zentrum Baden-Württemberg (BSZ): www.bsz-bw.de.

3 Verbundzentrale des Gemeinsamen Bibliotheksverbundes GBV (VZG): www.gbv.de.

4 Vgl. den Webauftritt zur Kooperation unter www.bszgbv.de. 
sorgt. Warum fanden sich aber zunächst „nur“ das BSZ und die VZG in einer Kooperation zur Modernisierung der Verbundstrukturen zusammen? Hätte dies nicht sofort und umfassend mit allen deutschen Verbundzentralen gelingen können und müssen?

Tatsächlich sprach in allererster Linie die Erwartungshaltung der Gutachtergremien der DFG gegen eine große Lösung mit allen deutschen Verbundzentralen. Denn in einem kompetitiven Antragsverfahren hatte sich die DFG für den CIB-Antrag und damit für das Ziel der Ablösung und der Aufgabe der deutschen Verbundkataloge zugunsten der Nutzung einer internationalen Metadatenbank, dem von OCLC betriebenen WorldCat, entschieden. Vor diesem Hintergrund war es den CIB-Projektteilnehmern nicht möglich, parallel zu dem von ihnen verfolgten und von der DFG geförderten „revolutionären“ Weg (Abschaffung der Verbundkataloge, stattdessen Katalogisierung im WorldCat) mit BSZ und VZG den „evolutionären“ Weg (Zusammenlegung und Modernisierung der Verbundkataloge) zu beschreiten.

Es gibt aber auch ganz praktische Gründe, warum das BSZ und die VZG in der Kooperation so gut zueinander passen: So nutzen BSZ und die VZG für ihre Kataloge die Software CBS der Firma OCLC, was hinsichtlich der in beiden Einrichtungen vorhandenen Strukturen und Kompetenzen natürlich von Vorteil war und ist. Daher konnte aus beiden Welten das Beste zusammengetragen und ein neues, gemeinsames CBS-System aufgesetzt werden. Neben BSZ und VZG setzt noch die hessische Verbundzentrale die CBS-Software als Verbundsystem ein. ${ }^{5}$ Allerdings hatte diese auch die Projektleitung des CIB-Vorhabens inne. Die Integration der hessischen Verbunddatenbank in das Vorhaben von BSZ und VZG war also zu Zeiten des CIB-Projekts für die hessische Verbundzentrale nicht zu leisten. BSZ und VZG sind aber weiterhin stark an der Zusammenarbeit mit weiteren Verbünden und Bibliotheken beim Einsatz des CBS-Systems interessiert.

Durch die institutionen- und verbundübergreifende Zusammenarbeit haben das BSZ und die VZG Synergien geschaffen und die Qualität ihrer Services verbessert. Auch gibt es eine Reihe von Arbeitsbereichen, in denen beide Partner Alleinstellungsmerkmale haben, die somit für die jeweils andere Verbundregion von hohem Interesse sind: Die entsprechenden Services werden einfach durch die Partnereinrichtung für die eigene Verbundregion miterbracht - und umgekehrt.

Zunächst haben daher beide Partner im Rahmen des 103. Bibliothekartags in Bremen ein gemeinsames Projekt mit dem Ziel der Verbesserung von Angebo-

5 Die Deutsche Nationalbibliothek (DNB) nutzt das CBS als Katalogisierungskomponente für ihr „Lokalsystem“. Auch die Zeitschriftendatenbank (ZDB) setzt auf das CBS-System. 
ten und Services bekannt gegeben. Dieses Vorhaben wurde anschließend mit den zuständigen Gremien diskutiert, Ziele formuliert und eine formelle Kooperationsvereinbarung verfasst. Die Vereinbarung, die auch von den zuständigen Ministerien mitgetragen und unterstützt wurde, konnte schließlich am 3. September 2015 im Osnabrück von den beiden Direktoren von BSZ und VZG unterzeichnet werden. Als Ziele wurden formuliert:

- Vereinigung der Verbundkataloge des BSZ und der VZG in einer Produktivumgebung

- Anpassung der technischen Strukturen der Pica-Systeme beider Verbünde

- Einführung eines gemeinsamen Internformats und einheitlicher Katalogisierungs- und Anwendungsrichtlinien

- Arbeitsteiliger Aufbau einer gemeinsam genutzten Infrastruktur für den Nachweis und die Administration von E-Ressourcen wie z. B. E-Books für die Verbundzentralen und ihre teilnehmenden Bibliotheken

- Aufbau und arbeitsteiliger Betrieb von Infrastruktur und Support für lokale Bibliothekssysteme und Discovery-Services

- Gemeinsame Bereitstellung eines Workflow-Systems zur retrospektiven Digitalisierung

- Einführung einer arbeitsteiligen Infrastruktur für Dienstleistungen zur Langzeitarchivierung

- Abstimmung von Personalentwicklung, Kompetenz- und Leistungssaufbau, um Redundanzen zu vermeiden und Synergien zu nutzen

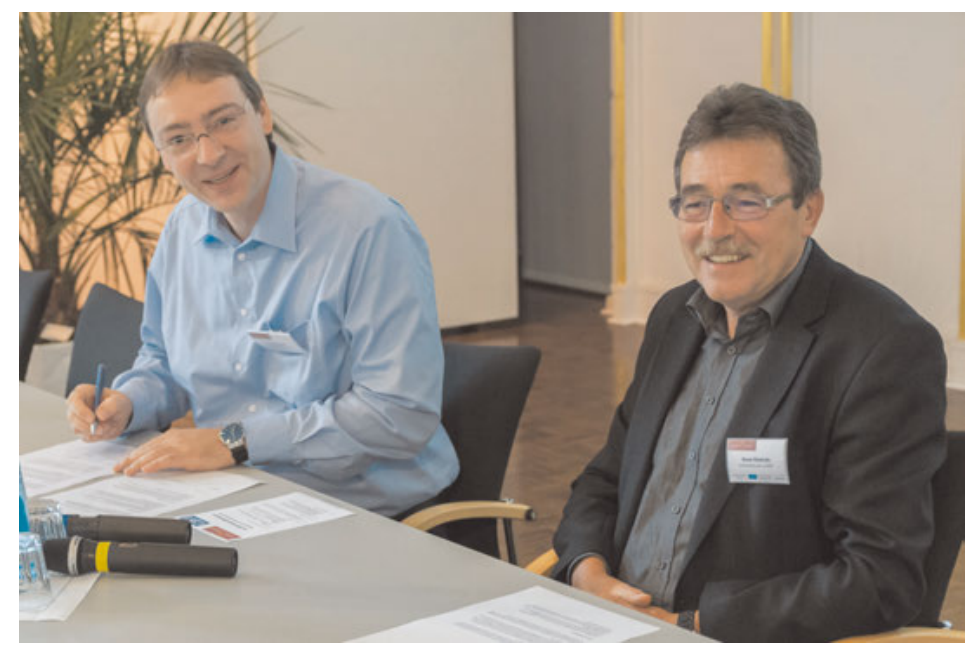

Abb. 1: Vertragsunterzeichnung am 3. September 2015 im Osnabrücker Schloss. 
Das BSZ und die VZG haben im Anschluss mit der inhaltlichen Arbeit an den vereinbarten Zielen begonnen und organisatorische Strukturen der Zusammenarbeit aufgebaut. Dabei sind stets zwei Fälle zu unterscheiden.

Fall 1: Das BSZ oder die VZG übernehmen Services für den jeweils anderen Partner und für dessen Verbundregion. Es werden keine redundanten technischen oder gedoppelte personelle Strukturen vorgehalten. Zum Beispiel sind die Programmierung und der Betrieb des EBM-Tools für die E-Book-Verwaltung von BSZ und VZG in der alleinigen Verantwortung des BSZ. Im Gegenzug betreut die VZG den so genannten E-Book-Pool, also eine Sammlung von Metadaten $\mathrm{zu}$ E-Book-Paketen, in Eigenverantwortung in Göttingen. Erst durch das Zusammenspiel beider Komponenten ergibt sich ein attraktiver Service für die Bibliotheken. Ein weiteres Beispiel ist die Aufteilung der Unterstützung von Lokalsystemen. Die VZG bietet aktuell die Unterstützung von LBS4 der Firma OCLC sowie perspektivisch der Open-Source-Lösung Folio an; das BSZ bietet aktuell die Unterstützung von aDIS/BMS, Koha und Alma an.

Fall 2: Das BSZ und die VZG teilen sich die Verantwortung bei gemeinsamen Services. Das bedeutet, dass Services hinsichtlich der personellen Strukturen arbeitsteilig an beiden Standorten organisiert werden. Ein wichtiges Beispiel ist die Zusammenlegung der beiden Verbunddatenbanken. Die neue gemeinsame Verbunddatenbank K10plus wird technisch in Göttingen betrieben. Das BSZ betreibt in Konstanz ein Spiegelsystem zur georedundanten Ausfallsicherheit und zur Lastverteilung für nachgeordnete lesende Anwendungen. Mitarbeiter beider Verbundzentralen arbeiten gemeinsam an Import, Export und Datenverarbeitung. Gemeinsam betreuen sie Technik und Formate; sie teilen dieselben Arbeitsumgebungen, dasselbe Wiki, dieselben Schulungsunterlagen etc.

\section{Angebotene Services}

Inhaltlich ist das Spektrum der bereits heute gemeinsam angebotenen Services groß:

- K10plus, der gemeinsame Verbundkatalog mit über 180 Millionen Bestandssätzen

- K10plus-Zentral, die kostenfrei nutzbare Discovery-Plattform mit mehr als 200 Millionen Datensätzen für ganz Deutschland

- EBM-Tool, das E-Book-Management-Tool zur Verwaltung von E-Book-Lizenzen und zur Beschleunigung der Verfügbarkeit in den lokalen Nachweissystemen 
- Digishelf, die Plattform für die Verarbeitung und die Präsentation digitalisierter Sammlungen und Bestände

\section{K10plus}

Im Herbst 2017 wurden die Datenbanken der beiden Verbundzentralen BSZ und VZG unter dem Namen K10plus erstmals zu einer gemeinsamen Datenbank mit über 180 Millionen Nachweisen fusioniert. Nach einem Test, einem eventuellen Neuladen der Daten und Schulungen werden die angeschlossenen Bibliotheken der beiden Verbundregionen ihre Bestände ab März 2019 in diesem Datenpool katalogisieren und Metadaten für ihre Lokalsysteme dort beziehen.

K10plus rationalisiert die wissenschaftliche Informationsversorgung durch seine breite und homogene Datenbasis. Zugleich eröffnen sich neue Perspektiven der Nachnutzung, beispielsweise zur Analyse und Steuerung der Informationsversorgung oder im Bereich der automatisierten Erschließung. K10plus sorgt für Standards und für die Bereitstellung von Normdaten aus dem Bibliotheks- und Wissenschaftsbereich.

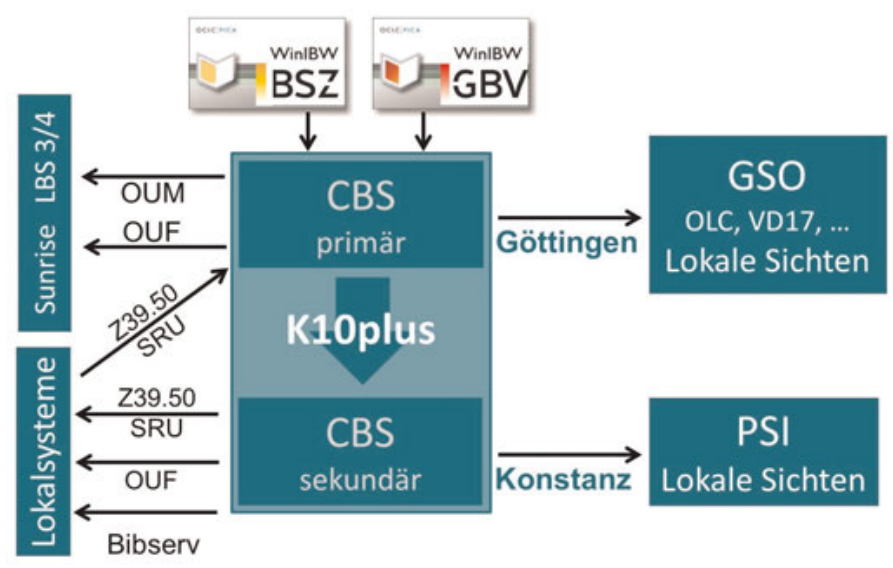

Abb. 2: Systemarchitektur K10plus.

K10plus agiert als Datenbroker und als Datendrehscheibe - national und international. Unterstützt werden regionale Nachweisinstrumente ebenso wie nationale und internationale Strukturen (bis hin zum WorldCat). Für die fachwissenschaftlichen Belange insbesondere DFG-geförderter Fachinformationsdienste 
werden spezielle auf die jeweilige Community zugeschnittene Services betrieben.

K10plus wird durch seine fachlich und zeitlich universelle inhaltliche Erstreckung, seine semantische Verlässlichkeit durch Standardisierung und Normdatenverwendung, durch die Anreicherung mit Provenienzen und Georeferenzen sowie durch die Navigationsmöglichkeit bis zum digitalen Exemplar selbst zum Gegenstand der Forschung: Auf Basis seiner Daten können semantische Recherchen der Bestände realisiert, geografische und historische Zusammenhänge der Publikationen visualisiert sowie über innovative Interaktionsformen neue Untersuchungsmethoden ermöglicht werden.

Ein wichtiger Aspekt von K10plus ist die Offenheit gegenüber Anwendungen auch über die typischen Werkzeuge des Bibliothekswesens hinaus: Mit webgestützten Standardschnittstellen, der Bereitstellung der Katalogisate als Linked Open Data unter einer Creative-Commons-Lizenz ist solchen Nachnutzungen der Weg geebnet. Wissenschaftliche Sammlungen in Archiven, Bibliotheken und Museen werden spartenübergreifend und zum Besten für die Forschung unterstützt. Forschungsdaten werden nachgewiesen und der direkte Zugriff auf vorhandene Datenrepositorien ermöglicht. K10plus ist offen für die Zusammenarbeit mit neuen Partnern, gerade aus der Wissenschaft, aber auch aus dem Bereich der bisherigen Verbundstrukturen sowie für einzelne Bibliotheken.

\section{K10plus-Zentral und K10plus-Discovery}

K10plus-Zentral wurde früher unter dem Namen GBV-Zentral betrieben. Es handelt sich um ein Index-Backend für bibliografische Daten mit weit mehr als 200 Millionen Datensätzen. Enthalten sind unter anderem der Gemeinsame Verbundkatalog (GVK), der Südwestdeutsche Bibliotheksverbund (SWB), der Verbundkatalog öffentlicher Bibliotheken (ÖVK), Online Contents (OLC), das Directory of Open Access Journals (DOAJ), PubMed/Medline, JSTOR, Springer E-Journals, die Datenbank TEMA Technik und Management der WTI-Frankfurt eG, Nationallizenzen sowie E-Books aus dem Projekt Gutenberg. Ergänzend eingebunden sind die Normdateien GND und RVK. Noch fehlende Inhalte werden durch die Einspielung weiterer Verlagsangebote (Elsevier, Wiley, De Gruyter, Hogrefe, Oxford University Press, SAGE usw.) und der Crossref-Daten ergänzt.

K10plus-Zentral ist ein Produkt der Community für die Community. Die Community entscheidet über die Inhalte, die Community behält die Hoheit über ihre Daten. Daher ist die Nutzung des Backend-Dienstes für alle wissenschaftlichen Bibliotheken in Deutschland kostenfrei. K10plus-Zentral basiert auf Solr 
und unterstützt alle Benutzeroberflächen, die Solr ansprechen können. Attraktiv ist, dass die Daten in MARC 21 bereitgestellt und bis hinunter auf Aufsatzebene durch Abgleich mit der ZDB mit Holdings, Lizenzen, Sachgruppen etc. angereichert werden. Der Index wird kooperativ mit den Bibliotheken gepflegt. Das „Geschäftsmodell“ ist communitybasiert: Bibliotheken melden bei der VZG oder dem BSZ ihren Bedarf an neuen Daten. Sie leisten Vorarbeit, indem sie Ansprechpartner bei den Verlagen identifizieren und die Rechtslage klären. Wenn möglich leisten sie auch Unterstützung bei der Normierung der Ausgangsdaten. Danach übernehmen die VZG und das BSZ alles Weitere.

Die VZG und das BSZ haben dieses Community-Modell bewusst gewählt, um sich bezüglich der Themen Datenhoheit und Datenqualität nicht in die Hände internationaler kommerziell orientierter Firmen zu begeben. K10plus-Zentral steht als kostenfreier Service allen öffentlich-rechtlichen Bibliotheken in Deutschland frei zur Nachnutzung zur Verfügung. Man darf gespannt sein, welche neuen Kooperationen sich über die Verbundgrenzen hinaus durch das gemeinsame Engagement an K10plus-Zentral entwickeln. Für die einzelne Einrichtung ist das Engagement im Community-Modell ein kalkulierbarer Aufwand bei gleichzeitig großem Mehrwert. Das Community-Modell stellt daher in vielen Fällen eine realistische Alternative zum Bezug kostenpflichtiger kommerzieller Mega-Indices dar.

Unter dem Namen K10plus-Discovery übernehmen die VZG und das BSZ das Hosting und den Betrieb individualisierter Suchoberflächen für einzelne Einrichtungen, dies allerdings kostenpflichtig. Datenräume und Zugriffsrechte werden dabei individuell auf die Bedarfe der Einrichtung hin konfiguriert. Zudem können auch Materialien, die nur für den Campuszugriff lizenziert sind, eingebunden werden.

\section{EBM-Tool}

Das EBM-Tool ermöglicht den Bibliotheken, über ein Webformular ihre Lizenzen $\mathrm{zu}$ verwalten und die Einspielung von Metadaten von E-Book-Paketen anzustoßen. Mit dem Tool wird die Verwaltung von E-Book-Metadaten, Paketen und Lizenzen verbessert und beschleunigt. Zurzeit stellen die Bibliotheken ihre Lizenzverwaltung schrittweise auf das EBM-Tool um. Mit Stand Herbst 2017 sind mehr als 300 Paketdefinitionen verfügbar, die bereits von mehr als 125 Bibliotheken genutzt werden.

Um bei Eintreffen einer Anforderung über das EBM-Tool rasch und automatisch reagieren $\mathrm{zu}$ können, betreiben BSZ und VZG im Hintergrund einen gemeinsamen E-Book-Pool, der auch für die Nutzung über WinIBW-Broadcast- 
Search zur Verfügung steht. Lässt sich eine EBM-Bestellung einem bereits im Pool vorhandenen Paket zuordnen, beschleunigt dies die Einspielung und die Verfügbarkeit von E-Books erheblich. Enthalten sind zurzeit ca. 1,4 Millionen Titel, unter anderem von De Gruyter, Springer, UTB, ProQuest, Forschungsberichte der TIB (Open Access) und vieles mehr. Metadaten von E-Book-Paketen aus dem Pool können auf Anfrage für Bibliotheken unabhängig von der Verbundzugehörigkeit in MARC 21 zur Verfügung gestellt werden. Daher eignet sich der Pool auch als Servicedienstleistung für Bibliotheken anderer Verbundregionen und eröffnet neue Perspektiven der verbundübergreifenden Kooperation.

\section{Digishelf}

Digishelf ist die gemeinsam betriebene digitale Bibliothek. Digishelf steht allen Einrichtungen aller Verbundregionen offen, die selbst keine Installation zur Bearbeitung, Verwaltung und Präsentation ihrer digitalisierten Sammlungen betreiben oder diese einrichten wollen. Von Vorteil ist die schlanke und kostengünstige Präsentation retrodigitalisierter Inhalte, welche alle nötigen Schnittstellen (auch die Ansicht im so genannten DFG-Viewer) bedient und ermöglicht. Über die Suchoberfläche von Digishelf werden alle Inhalte suchbar. Damit ergibt sich eine weite Sichtbarkeit für die teilnehmenden Bibliotheken und für ihre Sammlungen. Auf Wunsch können die Daten auch an die Deutsche Digitale Bibliothek (DDB) weitergeleitet werden. Das BSZ ist zudem Gründungsmitglied der DDB.

Die Möglichkeiten einzelner Einrichtungen, sich und ihre Sammlungen mit eigenem Logo und angepasstem Design in einem individuellen Suchraum zu präsentieren, wurden zum Jahresende 2017 deutlich erweitert. Anlass war die Überführung eines weiteren großen Datenbestands nach Digishelf, nämlich den Digitalisaten des Vereins „Bibliotheken der Regio Bodensee“. Im Digitalisierungsprojekt des Vereins haben sich 38 Bibliotheken der Region Bodensee zusammengeschlossen, um knapp 50 Zeitschriften und Jahrbücher aus BadenWürttemberg, Bayern, Liechtenstein, der Schweiz und Vorarlberg zu digitalisieren und in einem eigenen Webauftritt zu präsentieren. Auch dies ist ein hervorragendes Beispiel, wie Kooperation nicht nur über Verbundgrenzen, sondern über mehrere Länder hinweg erfolgreich organisiert und umgesetzt werden kann. 


\section{Wie organisiert man Kooperation?}

Da nicht die beiden Verbundorganisationen BSZ und VZG, sondern lediglich die technischen Systeme zusammengelegt und Services gemeinsam erbracht werden, bedarf es zur Wahrnehmung geteilter Verantwortung definierter Zuständigkeitsbereiche und klarer Kooperationsabsprachen. Die nötige Organisationsstruktur ist dabei schrittweise mit den Anforderungen gewachsen und ist auch weiterhin Anpassungen unterworfen. Dieser Prozess des Zusammenwachsens und der Veränderungen ist aus zwei Richtungen zu sehen: „Top-down“, also aus Sicht der Leitungs- und Entscheidungsgremien, und „Bottom-up“, also aus der Sicht der beteiligten Bibliotheken und ihrer Arbeitsgruppenstrukturen.

\section{Projektstruktur K10plus $\quad \mathrm{BSZ}^{-} \mathrm{GBV}$ Verbundkatalog für 10 Bundesländer, rbesitz und weitere Einrichtungen}

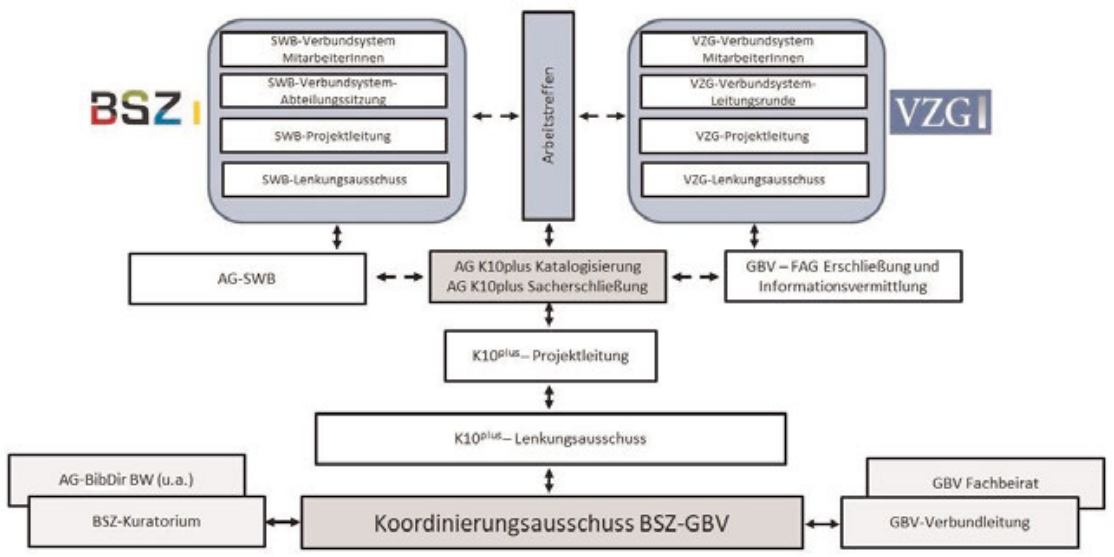

Abb. 3: Organisationsstruktur K10plus (Stand März 2018).

Für die Managementebene stellt der Koordinierungsausschuss BSZ-GBV das Bindeglied zwischen der Organisationsstruktur der Verbundzentralen einerseits und den Gremienstrukturen der Verbünde andererseits dar. Die jeweiligen Vertretungen der Ministerien der Sitzländer der Verbundzentralen, jeweils eine weitere Ministerialvertretung aus den beiden Verbundregionen, der bibliothekarische Vorsitz bzw. stellvertretende Vorsitz des jeweils zuständigen Verbundgremiums, eine weitere bibliothekarische Fachvertretung und die Leitungen des BSZ und der VZG bilden gemeinsam diesen Koordinierungsausschuss. Der Koordinierungsausschuss BSZ-GBV hat beratende und empfehlende Funktion 
sowohl für die beiden Verbundzentralen als auch für die zuständigen Gremien. Die Leitungs- und Aufsichtsfunktionen der jeweiligen Verbundgremien tangiert er nicht, gibt aber Orientierung und politische Unterstützung.

Auf der Ebene der Arbeitsgruppen ist die Einrichtung zweier übergreifender Arbeitsgruppen in Vorbereitung: Unter der Bezeichnung „AG K10plus Katalogisierung" wird eine Arbeitsgruppe für die Formalerschließung etabliert. Der Bereich der Sacherschließung wird in einer zweiten Arbeitsgruppe „AG K10plus Sacherschließung“ betreut. Die Arbeitsgruppen sollen sich paritätisch aus zehn bis zwölf Mitgliedern aus den Verbundregionen und den beiden Verbundzentralen zusammensetzen.

Auch wenn technische Aspekte bei der Kooperation des BSZ und der VZG eine große Rolle spielen, handelt es sich doch im Schwerpunkt um ein Organisationsvorhaben, welches Veränderung im Sinne eines Changemanagements gestalten muss. Denn verständlicherweise gibt es auch Unsicherheiten bei den Mitarbeitern. Die Veränderungen müssen erklärt, Ängsten begegnet und die großen Chancen der Zusammenarbeit dargelegt werden. Durch Arbeitsaufteilung und die entsprechenden Synergien gewinnen wir Raum, Neues zu denken und Innovation zu wagen. Gemeinsam sind wir stärker! Sichtbaren Ausdruck findet die Zusammenarbeit in dem gemeinsamen Logo für die gemeinschaftlich erbrachten Services.

\section{BSZ금 GBV}

Abb. 4: Logo für die gemeinsamen Projekte.

Faktisch bedeutet die Kooperation zunächst Mehrarbeit. Alles wird anstrengender und geht langsamer, weil der andere Partner die Dinge anders sieht und man erst zusammenfinden muss. Daher ist es wichtig, dass der neue gemeinsame Katalog K10plus wie geplant in Produktion geht. Alle Mitarbeiter im BSZ und in der VZG wollen die Ernte ihrer Arbeit nun bald einfahren. Erst dann wird Alltag einkehren, erst dann werden die Synergien für die Mitarbeiter, für die Bibliotheken und die Wissenschaftler spürbar und erfahrbar. 


\section{Zukunft}

Die oben angesprochenen Themenfelder bieten viel Potenzial für neue innovative Services. Diese sind, insbesondere im Bereich der spartenübergreifenden Zusammenarbeit zwischen Bibliotheken, Museen und Archiven, bereits in Arbeit.

\section{BSZ言GBV}

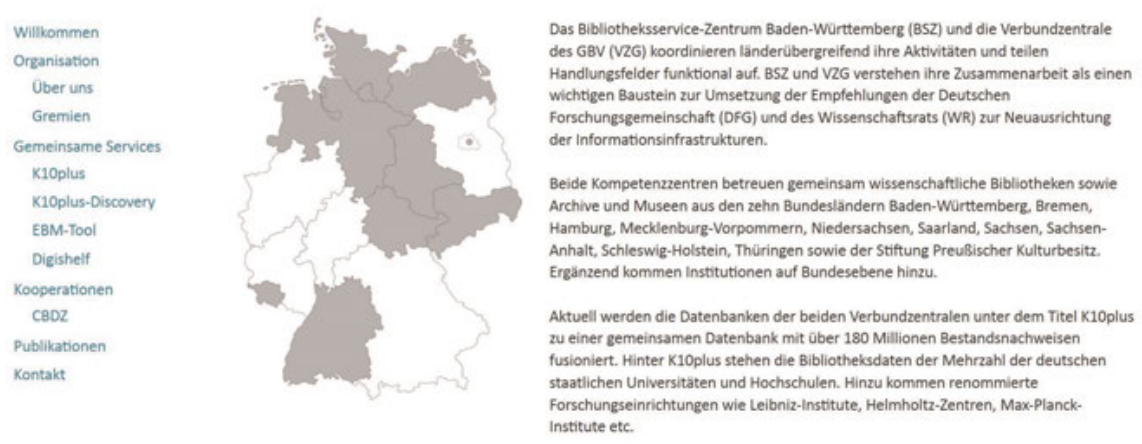

Abb. 5: Projektwebseite www.bszgbv.de (Erstinformationen zu den gemeinsamen Services).

Weiterhin wollen beide Verbundzentralen das Thema Electronic Resource Management (ERM) in Abstimmung mit den einschlägigen Projekten stärker betonen und dieses mit den Aktivitäten im E-Book-Bereich vernetzen. Schließlich wird auch das Thema eines neuen Bibliothekssystems mit Schwerpunkt bei den digitalen Ressourcen an Bedeutung gewinnen. Übergreifend im Rahmen der AGV werden das BSZ und die VZG das Thema Text- und Datamining gemeinsam adressieren.

Tradierte Verantwortlichkeiten und Rollen werden im Prozess der Digitalisierung der Informationsinfrastrukturen neu verhandelt. Die Herausforderungen der Zukunft sind nur im Rahmen von Kooperationen und in verteilter Verantwortung gestaltbar. Das BSZ und die VZG sind der festen Überzeugung, dass den anstehenden Aufgaben nur durch ein Kooperationsnetzwerk mit Universitäten und Hochschulen, starken wissenschaftlichen Bibliotheken, Forschungseinrichtungen und den Verbundzentralen erfolgreich begegnet werden kann. Hierfür setzen wir uns ein, hierfür werben wir um weitere Partner!

Weitere Informationen: www.bszgbv.de 